\title{
Diagnostic Value of Signal Peptide, CUB (Complement C1r/C1s, Uegf, and Bmp1), EGF (Epidermal Growth Factor)-Like Domain-Containing Protein 1 (SCUBE1) and Chemerin in Experimental Testicular Torsion
}

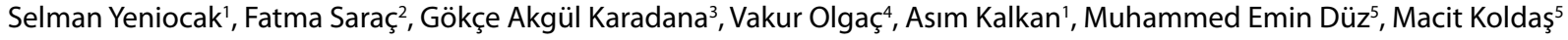 \\ 'Department of Emergency, University of Health Sciences, Haseki Training and Research Hospital, İstanbul, Turkey \\ 2Department of Pediatric Surgery, University of Health Sciences, Haseki Training and Research Hospital, ístanbul, Turkey \\ ${ }^{3}$ Department of Emergency Medicine, Koç University School of Medicine, İstanbul, Turkey \\ ${ }^{4}$ Department of Pathology, İstanbul University İstanbul School of Medicine, Oncology Institute, İstanbul, Turkey \\ ${ }^{5}$ Department of Clinical Chemistry, University of Health Sciences, Haseki Training and Research Hospital, İstanbul, Turkey
}

\begin{abstract}
Aim: The purpose of this experimental study was to investigate the potential diagnostic value of the platelet activation marker SCUBE1 [signal peptide, CUB (complement $\mathrm{C} 1 \mathrm{r} / \mathrm{C} 1 \mathrm{~s}$, Uegf, and Bmp1), and EGF (epidermal growth factor)-like domain-containing protein 1] and the adipocytokine chemerin in a prepubertal rat model of testicular torsion (TT).
\end{abstract}

Materials and Methods: Twenty-eight male rats were used for this study. They were randomly assigned into one of the four groups, each containing seven rats. No additional procedure other than a sham operation was performed on the control group (group IV). The other subjects comprised the torsion ischemia groups (groups I, II, and III). Blood specimens were collected after $30 \mathrm{~min}$ (group I), $2 \mathrm{~h}$ (group II), or $4 \mathrm{~h}$ (group III) using the intracardiac method. For group IV, which was the sham operation group, blood specimens were collected after $4 \mathrm{~h}$, and testis tissue specimens were extracted by orchiectomy for histopathological examination.

Results: No statistically significant change was determined in SCUBE1 levels of rats exposed to torsion. Also, no significant difference was observed between SCUBE1 levels of rats exposed to torsion and those of the control group. Statistically significant change was determined in chemerin levels during observation in rats exposed to torsion. This change was statistically significant between groups I and III. There was no statistically significant difference between chemerin levels of rats exposed to torsion (groups II II, and III) and those of the control group (group IV).

Conclusion: We observed no statistically significant differences when plasma SCUBE1 and chemerin levels of rats subjected to TT were compared with a control group in this study.

Keywords: Chemerin, experimental study, rat model, SCUBE1, testicular torsion

\section{Introduction}

Testicular torsion (TT) is a condition more commonly seen in children and it requires emergency surgery (1). Torsion of the spermatic cord occurs, and the spermatic blood vessels are obstructed. If detorsion is not performed within a suitable time frame, the testis undergoes necrosis (2). If the duration of ischemia exceeds $6 \mathrm{~h}$, permanent injury occurs in the germinal epithelium. Early identification of the effect of reperfusion in the germinal epithelium and surgical intervention are therefore highly important (3). Since there is no additional medical treatment to protect the testis against ischemia-reperfusion injury apart from surgical repairing, there are no sufficiently sensitive standard diagnostic or prognostic markers. There is therefore a need for novel, sensitive diagnostic or prognostic biochemical markers (4).

SCUBE1 [signal peptide, CUB (complement C1r/C1s, Uegf, and Bmp1), and EGF (epidermal growth factor)-like domain-containing protein 1] is a newly described cell surface protein (5) and is thought to be a novel platelet endothelial adhesion molecule. SCUBE1 accumulation has been determined immunohistochemically in the subendothelial matrix of advanced atherosclerotic lesions in humans. It is stored in platelet granules and is released from thrombin-activated platelet granules, where it enters the thrombus (6). Chemerin is one of the

Correspondence to: Selman Yeniocak e-mail: selmanyeniocakacil@hotmail.com 
several recently discovered adipokines released from adipose tissue. It is released in an inactive state, prochemerin, from adipose and other tissues. It is converted into chemerin by plasmin, factor XIla, and C1s belonging to the coagulation and fibrinolytic mechanism, neutrophil elastase and cathepsin $\mathrm{G}$ released from activated neutrophil granules and serine proteases released from mast cells by undergoing cleavage from the C-terminal end. Chemerin is a ligand for the G-protein-coupled chemokine-like receptor-1 (CMKLR1). CMKLR1 is primarily found in immune system cells such as dendritic cells, activated neutrophils, and macrophages $(7,8)$. Chemerin has a potential role in the immune response by performing a chemotactic function for cells releasing antigens in inflammation or tissue injury $(7,9)$.

Platelet and neutrophil activation occurs as a result of vascular endothelium injury following ischemia in TT (10). The limited studies in the literature have investigated the relation between platelet activation and testicular ischemia and have revealed that this may play an important role in the pathology of tissue injury. One experimental study reported that measurement of plasma SCUBE1 levels may be of diagnostic, therapeutic, or prognostic value but further studies are needed to confirm this (11). Our scan of the literature revealed no studies investigating the conversion into chemerin of inactive prochemerin and substances released from activated neutrophil granules and the diagnostic significance of serum levels thereof in TT. The purpose of this experimental study was to investigate the potential diagnostic value of the platelet activation marker SCUBE1 and the adipocytokine chemerin in a prepubertal rat model of TT.

\section{Materials and Methods}

\section{Study design and selection of subjects}

This experimental study was performed following receipt of ethical committee approval (No. 2015/67). Twenty-eight prepubertal male Wistar--Hannover rats with a mean age of 30 days and weighing 75-125 grams were used. The experimental animals were kept at a temperature of $22^{\circ} \mathrm{C} \pm 1^{\circ} \mathrm{C}$, in a 12-hour light:12-hour dark cycle, at a humidity level of $50 \%-60 \%$. Rats were given standard pellet chow and city drinking water until the day of the experiment. Rats were fasted for 12 hours preoperatively.

\section{Study protocol}

Twenty-eight male rats were used for this study. They were randomly assigned into one of the four groups, each containing seven rats.

Group I: $\quad(n=7)$; blood specimens taken 30 min after induction of torsion ischemia.

Group II: $\quad(\mathrm{n}=7)$; blood specimens taken $2 \mathrm{~h}$ after induction of torsion ischemia.

Group III: $(\mathrm{n}=7)$; blood specimens taken $4 \mathrm{~h}$ after induction of torsion ischemia.

Group IV: $\quad(n=7)$; control group, a sham operation group.

General anesthesia was induced before surgery with peritoneal injection of $10 \mathrm{mg} / \mathrm{kg}$ xylazine and $70 \mathrm{mg} / \mathrm{kg}$ ketamine hydrochloride. The skin of the scrotum was cleaned with $10 \%$ povidone iodine solution. A 2-cm vertical, cutaneous and subcutaneous, midline incision was made in the scrotum. The left testes in the scrotal space, together with the tunica vaginalis and spermatic cord, were separated from the gubernaculum with blunt dissection and removed/ exposed. No additional procedure other than a sham operation was performed on the control group (group IV). The other subjects comprised the torsion ischemia groups (groups I, II, and III). In these groups, an experimental extravaginal model of TT was established by rotating the left testes and their cord elements 720 degrees in a clockwise direction. The testis subjected to torsion was attached to the inner surface of the scrotum in two locations using 4/0 propylene sutures. Blood specimens were collected after $30 \mathrm{~min}$ (group I), $2 \mathrm{~h}$ (group II), or $4 \mathrm{~h}$ (group III) using the intracardiac method. Left testis tissues exposed to torsion were extracted with orchiectomy from all groups for histopathological evaluation. For group IV, the sham operation group, blood specimens were collected after $4 \mathrm{~h}$, and testis tissue specimens were extracted by orchiectomy for histopathological examination. The specimens obtained were frozen and stored.

\section{Laboratory analysis}

\section{SCUBE1 and chemerin measurement}

An ELISA kit (Catalog No. CSB-E16229r; Cusabio Biotech Co., Wuhan, Hubei, People's Republic of China) was used to determine SCUBE1 levels, following the manufacturer's instructions. Specimen absorbances were determined on a Biotek ELX800 (Biotek, Winooski, VT) microplate reader at a wavelength of $450 \mathrm{~nm}$. The results were expressed in $\mathrm{ng} / \mathrm{mL}$. The minimum detectable dose was $0.625 \mathrm{ng} /$ $\mathrm{mL}$.

An ELISA kit (Catalog No.CSB-EL019324RA; Cusabio Biotech Co.) was used to determine chemerin levels, following the manufacturer's instructions. Specimen absorbances were determined on a Biotek ELX800 (Biotek) microplate reader at a wavelength of $450 \mathrm{~nm}$. The results were expressed in $\mathrm{ng} / \mathrm{mL}$. The minimum detectable dose was $0.9 \mathrm{~g} / \mathrm{mL}$.

\section{Histological analysis and staining}

Testis tissue specimens were fixed in Bouin's solution for histopathological examination. Following routine procedures, tissues were fixed in paraffin blocks. Four-micrometer-thick sections were taken. Following staining with hematoxylin and eosin $(\mathrm{HE})$, these were examined under light microscope in line with the classification described by Cosentino et al. (12).

\section{Statistical analysis}

Statistical Package for the Social Sciences (SPSS Inc.; Chicago, IL, USA) 15.0 for Windows software was used for statistical analysis. Since parametric test conditions could not be established, comparisons of more than two independent groups were performed using the Kruskal-Wallis test. Subgroup analyzes were performed with the Mann-Whitney $U$ test and interpreted with Bonferroni correction. Dependent group analyzes were performed with the Wilcoxon test and interpreted with Bonferroni correction. Statistical alpha significance was set at $\mathrm{p}<0.05$.

\section{Results}

\section{Biochemical parameters}

Time-dependent biochemical parameter results by groups are shown in Table 1. No statistically significant change was determined in SCUBE1 levels of rats exposed to torsion. Also, no significant difference was observed between SCUBE1 levels of rats exposed to torsion and those of the control group $(p=0.422)$. 
Table 1. Time-dependent changes in SCUBE1 and chemerin

\begin{tabular}{|l|c|c|c|c|c|c|}
\hline & Group I & Group II & Group III & Group IV & \\
\cline { 2 - 7 } & Median $(95 \% \mathrm{Cl})$ & Median $(95 \% \mathrm{Cl})$ & Median $(95 \% \mathrm{Cl})$ & Median $(95 \% \mathrm{Cl})$ & $\mathrm{p}^{*}$ \\
\hline Rat SCUBE $(\mathrm{ng} / \mathrm{mL})$ & $19.1(4.4-54.4)$ & $22.0(-5.5-79.7)$ & $13.6(3.9-39.6)$ & $20.6(-10.4-90.5)$ & 0.422 \\
\hline Rat Chemerin $(\mathrm{ng} / \mathrm{L})$ & $23.6(16.2-57.9)$ & $19.1(17.2-21.5)$ & $15.9(14.2-17.3)$ & $16.2(12.8-24.9)$ & 0.004 \\
\hline $\mathrm{p}$ & Group I vs. II & Group I vs. III & Group I vs. IV & Group II vs. III & Group II vs. IV & Group III vs. IV \\
\hline $\begin{array}{l}\text { Abbreviation: Cl, confidence interval. } \\
\text { *Kruskal-Wallis test. } \\
\text { **Mann-Whitney U test: Bonferroni correction } \mathrm{p}<0.0083\end{array}$ & 0.003 & 0.032 & 0.011 & 0.199 & 0.605 \\
\end{tabular}

Statistically significant change was determined in chemerin levels during observation in rats exposed to torsion $(p=0.004)$. This change was statistically significant between groups I and III $(p=0.003)$. There was no statistically significant difference between chemerin levels of rats exposed to torsion (groups I, II, and III) and those of the control group (group IV) $(p=0.605)$.

Time-dependent torsion and control group SCUBE1 results are shown in Figure 1 and chemerin results in Figure 2.

\section{Histopathological examination}

Testis tissues from both the torsion and control groups were examined. Analysis of group I tissue of the testisrevealed minimal interstitial hemorrhage and changes in germ cell arrangement (grade 2) (Figure 3). Pronounced hemorrhage and irregular, occasionally necrotic and necrobiotic germ cells were observed in group II testis tissue (grades 2 and 3) (Figure 4). Irregular germ cells, cells shedding into the lumen, and occasional coagulation necrosis were observed in testis tissue from group III (grades 3 and 4) (Figure 5). Histopathology of testis tissues from the control group (group IV) was grade 1, and these findings were compatible with normal testis tissue both macro- and microscopically (Figure 6).

A comparison of histopathological grades of group testes subjected to torsion is given in Table 2. A statistically significant difference was observed between grades in the TT groups $(p<0.001)$. This difference was significant between all groups except for between groups I and II.

\section{Discussion}

No statistically significant change was determined in plasma SCUBE1 levels of rats subjected to torsion at $30 \mathrm{~min}$ and 2 or $4 \mathrm{~h}$. At the same time, no significant difference was determined in SCUBE1 levels of rats subjected to torsion compared to control group levels. No significant correlation was determined between SCUBE1 levels in TT and histopathological scoring.

SCUBE1 is a novel potential marker of platelet activation (13). This cell surface protein is largely stored in the alpha granules of the platelets. Significant amounts of SCUBE1 are secreted with platelet stimulation (14). Dai et al. (6) reported a significant increase in plasma SCUBE1 concentration acute ischemic stroke (AIS) and acute coronary syndrome (ACS), in which platelet activation is involved, and an important increase in coronary artery disease. In an experimental study, Turkmen et al. observed a sudden increase in SCUBE1 levels 2 $\mathrm{h}$ after acute mesenteric ischemia (AMI) and a pronounced increased in SCUBE1 levels $6 \mathrm{~h}$ after AMI (15).

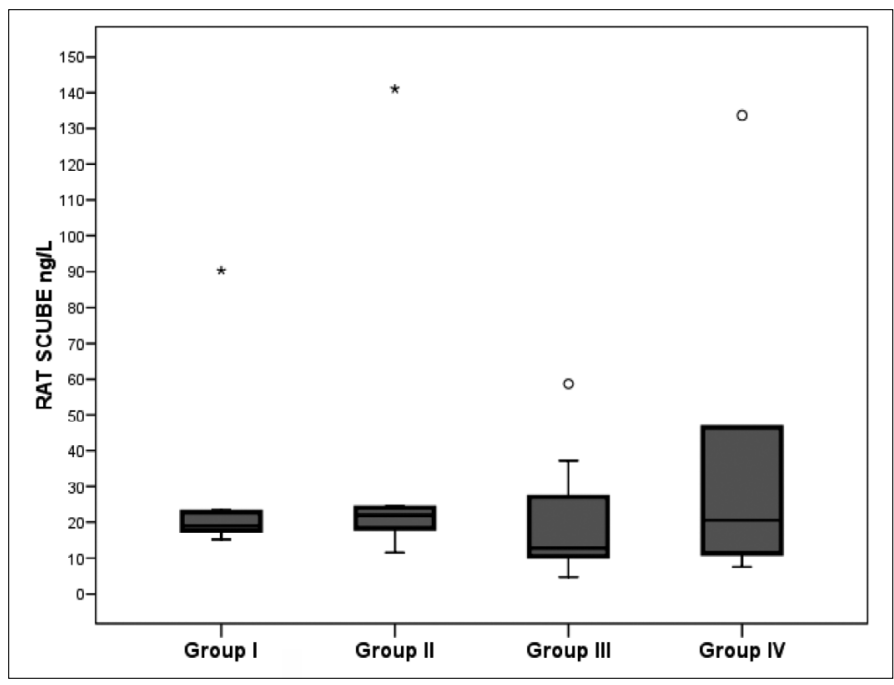

Figure 1. Time-dependent SCUBE1 levels in the torsion (groups I, II and III) and control group (Group IV)

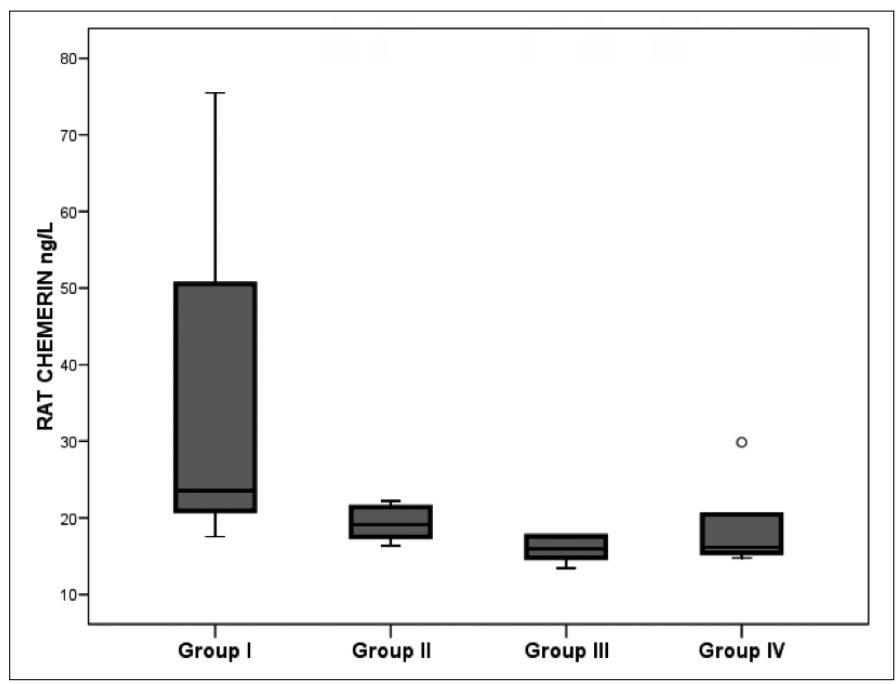

Figure 2. Time-dependent chemerin levels in the torsion (groups I, II and III) and control group (Group IV).

In one study investigating the role of platelet activator factor (PAF) in testicular ischemia, the PAF antagonist CV-6209 was used before torsion; significantly, less ischemic injury was observed compared to the untreated group (16). In another study, Orhan et al. (3) used the PAF antagonist EGB-761 Gingko biloba after torsion and re- 


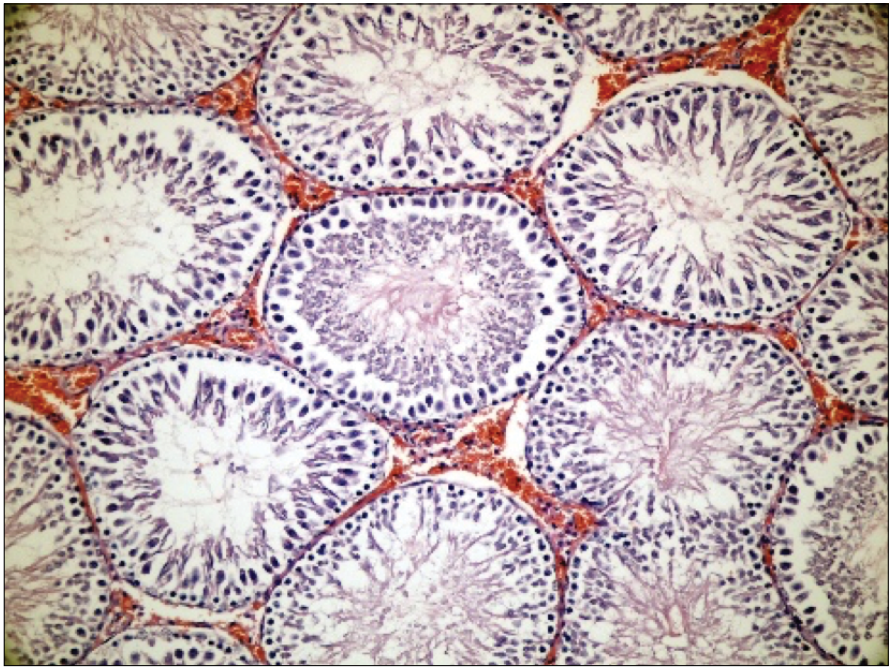

Figure 3. Minimal interstitial hemorrhage and changes in germ cell arrangement in the 30-min torsion group (Grade 2) (H\&E X200).

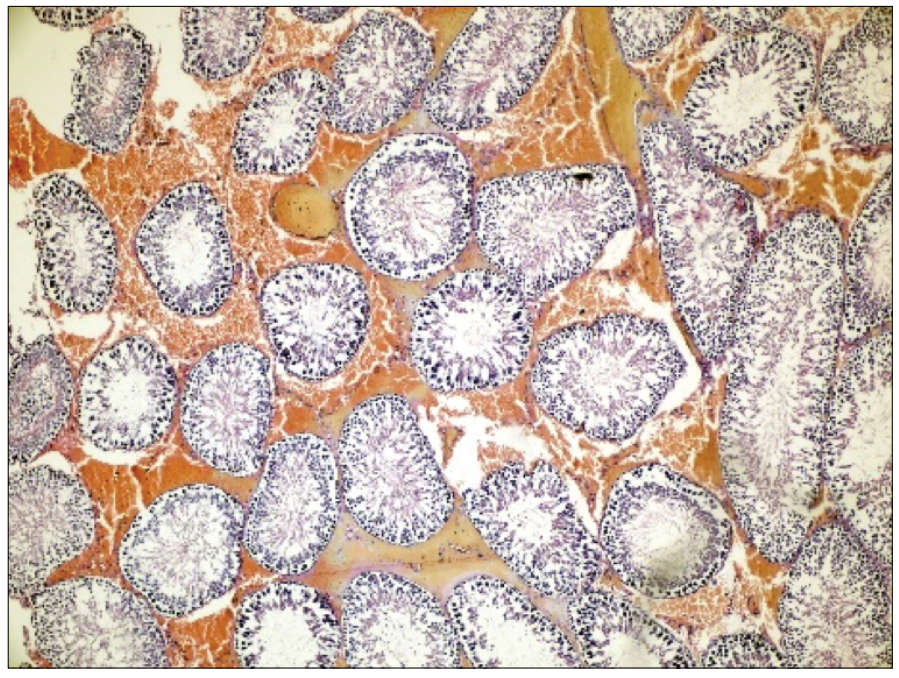

Figure 4. Pronounced hemorrhage and irregular, occasionally necrotic and necrobiotic germ cells in the 2-h torsion group (Grade $2-3$ ). (H\&E X100).

Table 2. Comparison of the histopathological grades of the groups

\begin{tabular}{|c|c|c|c|c|c|c|}
\hline & & Group I & Group II & Group III & Group IV & $\mathbf{p}^{*}$ \\
\hline \multirow[t]{3}{*}{ Torsion ever } & Mean \pm SD & $2.00 \pm 0.58$ & $2.29 \pm 0.49$ & $3.14 \pm 0.38$ & $1.00 \pm 0.00$ & \multirow[b]{2}{*}{$<0.001$} \\
\hline & Median & 2 & 2 & 3 & 1 & \\
\hline & Group I vs. II & Group I vs. III & Group I vs. IV & Group II vs. III & Group II vs. IV & Group III vs. IV \\
\hline$p$ & 0.334 & 0.003 & 0.002 & 0.007 & 0.001 & $<0.001$ \\
\hline
\end{tabular}

ported a significant decrease in ischemic injury induced by torsion in the contralateral testis. PAF is a phospholipid mediator with powerful biological effects. It activates a broad cell group including monocytes, mast cells, and polymorphonuclear leukocytes, and particular-

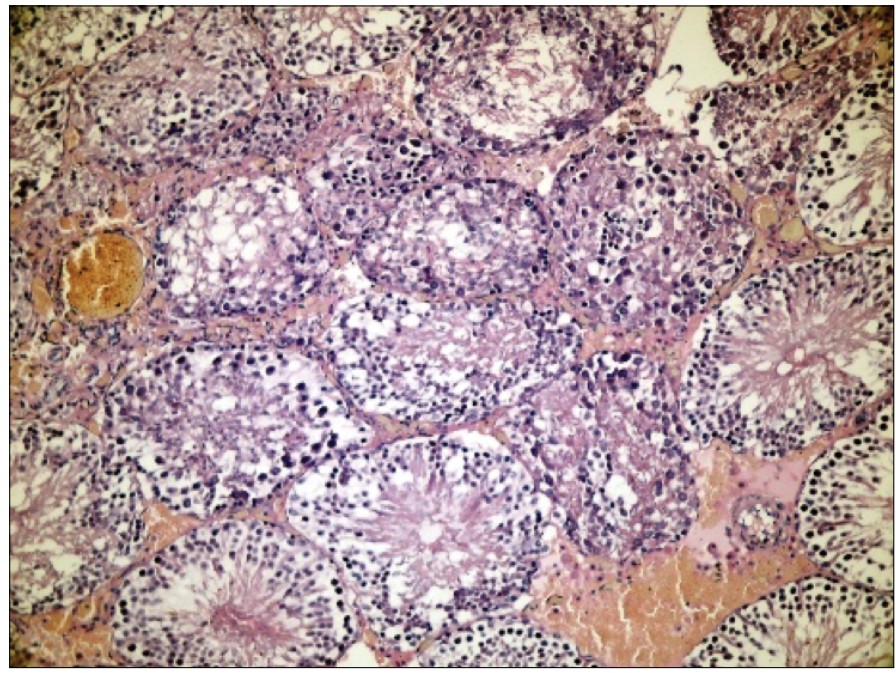

Figure 5. Irregular germ cells, cells shedding into the lumen and occasional coagulation necrosis in the 4-h torsion group (Grade $3-4$ ) (H\&E X200)

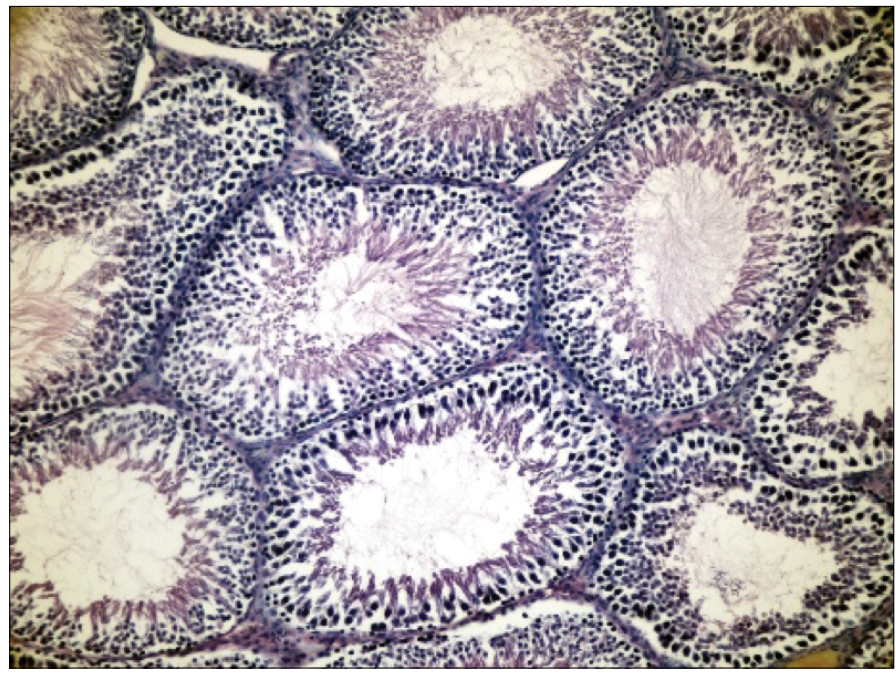

Figure 6. Testis tissue with a normal appearance in the control group (Grade 1) (H\&E X 200). ly platelets (17). No statistically significant change was determined in the SCUBE1 levels of the torsion group in our study. No significant difference was determined between torsion groups' SCUBE1 levels and those of the control group. In an experimental study of adult rats, 
Türedi et al. (11) compared plasma SCUBE1 levels in groups consisting of six rats and exposed to TT at $2 \mathrm{~h}$ and $4 \mathrm{~h}$ with control group levels at 2 and $4 \mathrm{~h}$. A significant elevation in SCUBE1 levels was determined $4 \mathrm{~h}$ after testicular ischemia. In our study, we elected to use prepubertal rats rather than adults, as TT is more common in very young males. We measured plasma SCUBE1 levels in groups containing seven prepubertal rats each exposed to TT at $30 \mathrm{~min}, 2 \mathrm{~h}$, and 4 $\mathrm{h}$ and levels in the control group at $4 \mathrm{~h}$. No significant change was determined in plasma SCUBE1 levels of the rats exposed to torsion. In addition, there was no statistically significant difference between TT group plasma SCUBE1 levels and those of the control group. Türedi et al. (11) reported a significant correlation between TT SCUBE1 levels and histopathological scores. In our study, however, no significant correlation was observed between TT SCUBE1 levels and histopathological scores. In contrast to Türedi et al.'s (11) study, which reported that measurement of plasma SCUBE1 levels could be of diagnostic, therapeutic, or prognostic values in TT, we concluded that these are of no diagnostic value.

Complement $3 \mathrm{a}$ and complement $5 \mathrm{a}$, chemotactic factors that appear in the ischemic area in TT, cause neutrophils to migrate to the region (10). Prochemerin is converted into chemerin with cathepsin $\mathrm{G}$ and neutrophil elastase released from activated neutrophil granules. Chemerin performs a chemotactic function for cells producing antibodies in inflammation or tissue injury. CMKLR1 stimulates chemotaxis of expressing dendritic cells macrophages and enables these cells to be directed to the area of inflammation $(7,9)$. Goralski et al. (18) reported that chemerin and CMKLR1 expression in epididymal white adipose tissue was twice as high compared to stromal vascular fraction.

Kadoglou et al. (19) reported an in increase in chemerin levels in a study of patients with acute myocardial infarction (AMI). Ji et al. (20) reported that plasma chemerin levels increased in patients with ACS, with the exception of stable angina pectoris. In a clinical study, Zhao et al. (21) reported an increase in plasma chemerin levels with AIS and carotid artery atherosclerosis. Chemerin levels in atherosclerosis increased in correlation with plaque instability and were higher in AIS. Zhao et al. (21) examined serum chemerin levels in patients with AIS and carotid artery atherosclerosis and investigated whether chemerin elevation might be a possible risk factor for these diseases. In the same clinical study, chemerin levels increased in correlation with plaque instability in carotid artery atherosclerosis and were higher in AIS. The authors concluded that chemerin levels might play an indicative role in monitoring the development of atherosclerosis and cerebral infarction.

In our study, chemerin levels decreased as the duration of torsion ischemia increased. We attributed this to the development of torsion ischemia not being vascular plaque--dependent. In an experimental study of intestinal ischemia-reperfusion injury induced in mice by blocking the mesenteric artery, Zhu et al. (22) reported a significant increase in plasma chemerin levels after 60-min reperfusion following 60-min ischemia. A statistically significant change was determined in chemerin levels in rats subjected to torsion in our study. This was statistically significant between the 2-h (group II) and 4-h (group III) TT groups. However, no statistically significant difference was determined between the plasma chemerin levels of torsion groups and those of the control group. At histopathological scoring, there was a statistically significant difference between the TT group grades. However, there was no significant difference between group I and II TT grades. Also, there was no significant correlation determined between TT plasma chemerin levels and histopathological scores. The reason for the absence of a statistically significant difference with the control group, although chemerin levels decreased, is that vascular coagulation did not occur during the TT process. Our study did not investigate chemerin levels after $4 \mathrm{~h}$ following torsion ischemia. We therefore think that wider studies are now needed in human patients.

\section{Study limitations}

Prepubertal rats were used in this study, as TT is more commonly seen in very young males. Hematological status in prepubertal rats may differ in various ways compared to adult rats and in humans. At the same time, the experimental model may not exactly mimic typical cases of TT seen in clinical practice. We measured chemerin and SCUBE1 levels by exposing rats to experimental torsion for $30 \mathrm{~min}$ and 2 and $4 \mathrm{~h}$. Plasma levels of these markers were not measured in the long-term after torsion ischemia. While reperfusion injury following detorsion is known to be as significant a factor as ischemia in patients with TT, detorsion was not applied in this experimental model.

\section{Conclusion}

We observed no statistically significant differences when plasma SCUBE1 and chemerin levels of rats subjected to TT were compared with a control group in this study.

Ethics Committee Approval: Ethics committee approval was received for this study from the ethics committee of Istanbul University Animal Experiments Local Ethical Committee (No. 2015/67).

Peer-review: Externally peer-reviewed.

Conflict of Interest: No conflict of interest was declared by the authors.

Financial Disclosure: We thank the Haseki Research and Training Hospital thesis and academic activities information and supervision commission for its support for this project.

\section{References}

1. Kutlu O, Mentese A, Turkmen S, Turedi S, Gunduz A, Yulug E, et al. Investigation of the possibility of using ischemia-modified albumin in testicular torsion: an experimental study. Fertil Steril 2011; 95: 1333-7. [CrossRef]

2. Ciftci AO, Senocak ME, Cahit Tanyel F, Buyukpamukcu N. Clinical predictors for differential diagnosis of acute scrotum. Eur J Pediatr Surg 2004; 14: 333-8. [CrossRef]

3. Orhan I, Hayit H, Duksal I, Ozercan HI, Firdolas F, Semercioz A. PAF antagonist protective effect on ischemic damage of contralateral testes in unilateral testes torsion. Turk J Urol 2004; 30: 11-6.

4. Beheshtian A, Salmasi AH, Payabvash S, Kiumehr S, Ghazinezami B, Rahimpour $S$, et al. Protective effects of sildenafil administration on testicular torsion/detorsion damage in rats. World J Urol 2008; 26: 197-202. [CrossRef]

5. Tu CF, Yan YT, Wu SY, Djoko B, Tsai MT, Cheng CJ, et al. Domain and functional analysis of a novel platelet-endothelial cell surface protein, SCUBE1. J Biol Chem 2008; 283: 12478-88. [CrossRef]

6. Dai DF, Thajeb P, Tu CF, Chiang FT, Chen $\mathrm{CH}$, Yang RB, et al. Plasma concentration of SCUBE1, a novel platelet protein, is elevated in patients with acute coronary syndrome and ischemic stroke. J Am Coll Cardiol 2008; 51: 2173-80. [CrossRef] 
7. Wittamer V, Franssen JD, Vulcano M, Mirjolet JF, Le Poul E, Migeotte I, et al. Specific Recruitment of Antigen-presenting cells by Chemerin, a Novel Processed Ligand from human inflammatory fluids J Exp Med 2003; 198: 977-85. [CrossRef]

8. Meder W, Wendland M, Busmann A, Kutzleb C, Spodsberg N, John H, et al. Characterization of human circulating TIG2 as a ligand for the orphan receptor ChemR23. FEBS Lett 2003; 555: 495-9. [CrossRef]

9. Zabel BA, Silverio A, Butcher EC. Chemokine-Like Receptor 1 Expression and Chemerin-Directed Chemotaxis Distinguish Plasmacytoid from Myeloid Dendritic Cells in Human Blood. J Immunol 2005; 174: 244-51. [CrossRef]

10. Welbourn CR, Goldman G, Paterson IS, Valeri CR, Shepro D, Hechtman HB. Pathophysiology of ischemia reperfusion injury: central role of the neutrophil. Br J Surg 1991; 78: 651-5. [CrossRef]

11. Turedi S, Tatli O, Alver A, Karaguzel E, Karaca Y, Turkmen S, et al. The diagnostic value of plasma SCUBE1, a novel biomarker of platelet activation, in Testicular Torsion: A Randomized, Controlled, Experimental Study. Urology 2015; 86: 516-20. [CrossRef]

12. Cosentino MJ, Nishida M, Rabinowitz R, Cockett AT. Histopathology of prepubertal rat testes subjected to various durations of spermatic cord torsion. J Androl 1986; 7: 23-31. [CrossRef]

13. Menteşe A, Yilmaz G, Sümer A, Arslan M, Karahan SC, Köksal I. The diagnostic and prognostic significance of SCUBE1 levels in Crimean -Congo hemorrhagic fever. Int J Infect Dis 2013; 7: 1042-5. [CrossRef]

14. Tu CF, Su YH, Huang YN, Tsai MT, Li LT, Chen YL, et al. Localization and characterization of a novel secreted protein SCUBE1 in human platelets. Cardiovasc Res 2006; 71: 486-95. [CrossRef]
15. Turkmen S, Mentese S, Mentese A, Sumer AU, Saglam K, Yulug E, et al. The value of signal peptide-CUB-EGF domain-containing protein 1 and oxidative stress parameters in the diagnosis of acute mesenteric ischemia. Acad Emerg Med 2013; 20: 257-64. [CrossRef]

16. Palmer JS, Cromie WJ, Plzak LF, Leff AR. A platelet activating factor antagonist attenuates the effects of testicular ischemia. J Urol 1997; 158: 1186-90. [CrossRef]

17. Walterscheid JP, Ullrich SE, Nghiem DX. Platelet-activating factor, a molecular sensor for cellular damage, activates systemic immune suppression. J Exp Med 2002; 195: 171-9. [CrossRef]

18. Goralski KB, McCarthy TC, Hanniman EA, Zabel BA, Butcher EC, Parlee SD, et al. Chemerin, a novel adipokine that regulates adipogenesis and adipocyte metabolism. J Biol Chem 2007; 282: 28175-88. [CrossRef]

19. Kadoglou NP, Tahmatzidis DK, Giannakoulas C, Kapelouzou A, Gkontopoulos A, Parissis J, et al. Serum levels of novel adipokines, omentin-1 and chemerin, in patients with acute myocardial infarction: KOZANI STUDY. J Cardiovasc Med (Hagerstown) 2015; 16: 341-6. [CrossRef]

20. Ji Q, Lin Y, Liang Z, Yu K, Liu Y, Fang Z, et al. Chemerin is a novel biomarker of acute coronary syndrome but not of stable angina pectoris. Cardiovascular Diabetology 2014; 13: 145. [CrossRef]

21. Zhao D, Bi G, Feng J, Huang R, Chen X. Association of Serum Chemerin Levels with Acute Ischemic Stroke and Carotid Artery Atherosclerosis in a Chinese Population. Med Sci Monit 2015; 21: 3121-8. [CrossRef]

22. Zhu QK, He GZ, Li HL. Effect of Intestinal Ischemia-reperfusion Injury on the Expression of Chemerin in Mice. Zhongguo Yi Xue Ke Xue Yuan Xue Bao 2015; 37: 440-5. 